\title{
A Linear Beacon System Featuring an Internal Deoxyguanine Quencher Allows Highly Selective Detection of Single Base Mismatches
}

\author{
Young-Ae Lee and Gil Tae Hwang* \\ Department of Chemistry, Kyungpook National University, Daegu 702-701, Korea. *E-mail: giltae@knu.ac.kr \\ Received May 12, 2010, Accepted May 27, 2010
}

\begin{abstract}
The fluorescence intensity of a single-stranded oligonucleotide containing a fluorene-labeled deoxyuridine $\left(\mathbf{U}^{\mathrm{Fl}}\right)$ unit increases by only 1.5 -fold upon formation of its perfectly matched duplex. To increase the fluorescence signal during hybridization, we positioned a quencher strand containing a deoxyguanine $(\mathrm{dG})$ nucleobase, functioning as an internal quencher, opposite to the $\mathbf{U}^{\mathrm{Fl}}$ unit to reduce the intrinsic fluorescence upon hybridization with a probe. From an investigation of the optimal length of the quencher strand and the effect of the neighboring base sequence, we found that a short strand (five-nucleotide) containing all natural nucleotides and $\mathrm{dG}$ as an internal quencher was effective at reducing the intrinsic fluorescence of a linear beacon; it also exhibited high total discrimination factors for the formation of perfectly matched and single base-mismatched duplexes. Such assays that function based on clear changes in fluorescence in response to single-base nucleotide mutations would be useful tools for accelerating diagnoses related to various diseases.
\end{abstract}

Key Words: DNA, Fluorene, SNP, Molecular beacon, Deoxyguanine

\section{Introduction}

Typical molecular beacons (MBs) are single-stranded oligodeoxynucleotides (ODNs) that, unless bound to their targets, possess a hairpin conformation. The 5' end of such an MB features a fluorescent dye; a quencher dye is covalently attached to the $3^{\prime}$ end. Therefore, when the beacon is not bound to its target, the hairpin structure positions the fluorophore and quencher in close proximity and no fluorescence is observed. ${ }^{1} \mathrm{Se}-$ veral methods for the detection of single-nucleotide polymorphisms (SNPs) have been established, based on fluorescence responses upon allele-specific hybridization, ${ }^{2}$ enzymatic reactions, ${ }^{3}$ DNA chips, ${ }^{4}$ and nanoparticles. ${ }^{5}$

Recently, we reported a new single-stranded, quencher-free MB for SNP typing; it consisted of only a single fluorophore (a fluorene-labeled deoxyuridine, $\mathbf{U}^{\mathrm{Fl}}$ ) positioned in the hairpin loop. ${ }^{6}$ When this MB undergoes DNA hybridization, it can discriminate between fully matched and single base-mismatched sequences. When the fluorene unit is intercalated (located) within a DNA duplex in the absence of base pairing (mismatched duplex), the fluorescence of the $\mathbf{U}^{\mathbf{F l}}$ unit is quenched as a result of photoinduced charge transfer originating from interactions with its neighboring nucleobases. The major drawback of this quencher-free MB system is its poor detection sensitivity, due to its intrinsic emission intensity. In this study, we employed a completely natural strand containing a deoxyguanine (dG) nucleobase, known to be an internal quenching base because of its good electron-donating property, ${ }^{7-9}$ to minimize the intrinsic fluorescence of our MB system (Figure 1). We investigated several parameters to improve the performance of the quencher, including its sequence dependency and neighboring base effects. We observed a clear improvement in the detection sensitivity for the perfectly matched target when using this internal quencher-probe system. Indeed, our quencher-free linear beacon probe appears to be a useful tool for SNP typing.

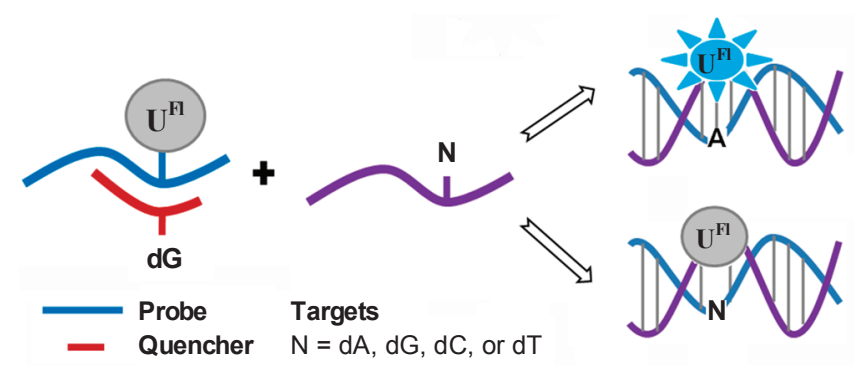

Figure 1. A linear beacon system designed in this study.

\section{Experimental Section}

Synthesis of oligonucleotides. A fluorene-labeled phosphoramidite was synthesized as described. ${ }^{6}$ ODNs were prepared using the $\beta$-cyanoethylphosphoramidite method on controlled pore glass supports $(1 \mu \mathrm{mol})$ with a POLYGEN Professional 12-Column DNA synthesizer and standard methods. ${ }^{10}$ After automated synthesis, the oligonucleotides were cleaved from the solid support and deprotected through treatment with 30\% aqueous $\mathrm{NH}_{4} \mathrm{OH}(1.0 \mathrm{~mL})$ for $10 \mathrm{~h}$ at $55^{\circ} \mathrm{C}$. The crude products from the automated ODN synthesis were lyophilized and diluted with distilled water $(1 \mathrm{~mL})$; they were then purified using highperformance liquid chromatography (HPLC; Grace VyDAC ${ }^{\mathrm{TM}}$ C18 column, $250 \times 10 \mathrm{~mm}$; pore size: $120 \AA$ ). The HPLC mobile phase was held isocratically for $10 \mathrm{~min}$ using $5 \%$ acetonitrile/ 0.1 $\mathrm{M}$ triethylammonium acetate (TEAA; $\mathrm{pH} 7.0$ ) at a flow rate of $2.5 \mathrm{~mL} / \mathrm{min}$. The gradient was then increased linearly over 10 min from $5 \%$ acetonitrile/ $0.1 \mathrm{M}$ TEAA to $50 \%$ acetonitrile/ 0.1 $\mathrm{M}$ TEAA at the same flow rate. The fractions containing the purified ODN were pooled and lyophilized. Aqueous $\mathrm{AcOH}$ (80\%) was added to the ODN; after standing for 30 min at ambient temperature, the $\mathrm{AcOH}$ was evaporated under reduced pressure. The residue was diluted with water $(1 \mathrm{~mL})$ and then the 


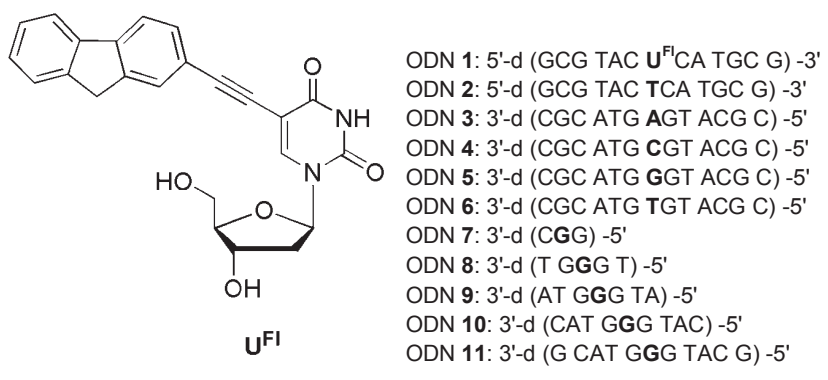

Figure 2. DNA sequences used in this study.

solution was purified through HPLC under the same conditions described above. The concentrations of the ODNs were determined through measurements of UV-vis absorptions. For characterization, matrix-assisted laser desorption ionization timeof-flight (MALDI-TOF) mass spectra of the ODNs were recorded using a PE Biosystems Voyager System 4095 spectrometer operated in the positive-ion mode with a 1:1 mixture of 3-hydroxypicolinic $\operatorname{acid}(0.35 \mathrm{M})$ and ammonium citrate $(0.1 \mathrm{M})$ as the matrix; the accelerating voltage was $25 \mathrm{kV}$.

Melting temperature $\left(T_{\mathrm{m}}\right)$ measurements. The unnatural and natural nucleosides were incorporated into the complementary ODNs 5'-d(GCGTACXCATGCG)-3' and 3'-d(CGCATGNGTACGC)-5' at the positions labeled $\mathbf{X}$ and $\mathbf{N}$. In UV melting experiments, the absorption of each sample $[1.5 \mu \mathrm{M}$ duplex in 100 $\mathrm{mM}$ Tris- $\mathrm{HCl}$ buffer ( $\mathrm{pH} 8.0$ ) containing $1 \mathrm{mM} \mathrm{MgCl}_{2}$ ] was monitored at $260 \mathrm{~nm}$ from 5 to $90{ }^{\circ} \mathrm{C}$ at a heating rate of $1{ }^{\circ} \mathrm{C} / \mathrm{min}$, using a Cary $300 \mathrm{Bio}$ UV-vis spectrophotometer. Melting temperatures were determined using a derivative method and Cary Win UV thermal application software.

UV and fluorescence measurements. ODN solutions were prepared as described for the $T_{\mathrm{m}}$ measurements. Fluorescence spectra were obtained using a PTI Fluorescence System spectrofluorophotometer, with a cell path length of $1 \mathrm{~cm}$ and excitation at $340 \mathrm{~nm}$. The excitation and emission bandwidth was $1 \mathrm{~nm}$.

\section{Results and Discussion}

Incorporation of the fluorene-labeled deoxyuridine $\mathbf{U}^{\mathbf{F l}}$ into the central position of the ODN 1 was effected using standard protocols of automated DNA synthesis (Figure 2). ${ }^{6,10}$ The fluorescence properties of fluorophore-containing DNA are strongly dependent on the electron injection and transfer properties of the flanking bases; indeed, flanking cytosine and thymine nucleobases are efficient quenchers for fluorophore emission in single-stranded oligonucleotides. ${ }^{11,12}$ Therefore, we positioned two deoxycytosine (dC) units as the bases flanking the $\mathbf{U}^{\mathrm{Fl}}$ unit.

To evaluate the thermodynamic stability and selectivity of $\mathbf{U}^{\mathrm{Fl}}$ in duplex DNA, we incorporated $\mathbf{U}^{\mathrm{Fl}}$ and natural nucleosides into the complementary oligonucleotides 5'-d(GCGTACXCATGCG)-3' and 3'-d(CGCATGNGTACGC)-5' at the positions labeled $\mathbf{X}\left(\mathbf{U}^{\mathrm{H}}, \mathrm{ODN} \mathbf{1}\right.$; dT, ODN 2) and $\mathbf{N}(\mathrm{dA}, \mathrm{dC}, \mathrm{dG}$, and dT; ODNs 3-6, respectively). The melting temperature $\left(T_{\mathrm{m}}\right)$ of each duplex, a measure of the base pair stability, was determined through thermal denaturation experiments (Table 1). The duplex $\mathbf{1 \cdot 3}$ was $5.2^{\circ} \mathrm{C}$ less stable than the natural duplex $\mathbf{2 \cdot 3}$ in the same sequence context, but it was significantly $\left(5.0-9.6{ }^{\circ} \mathrm{C}\right)$ more

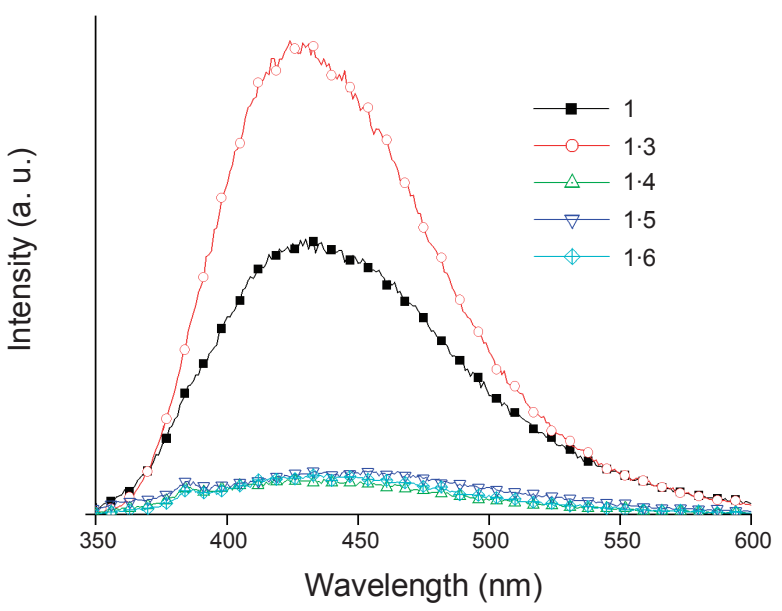

Figure 3. Fluorescence spectra of the single-stranded ODN 1 and the duplexes formed between $\mathbf{1}$ and the targets 3-6. All spectra were recorded at $20^{\circ} \mathrm{C}$ using $1.5 \mu \mathrm{M}$ solutions of the samples in $10 \mathrm{mM}$ Tris- $\mathrm{HCl}$ buffer ( $\mathrm{pH} 7.2 ; 100 \mathrm{mM} \mathrm{NaCl}, 20 \mathrm{mM} \mathrm{MgCl}_{2}$ ), with irradiation at 340 $\mathrm{nm}$.

Table 1. Thermal melting temperatures of modified and unmodified duplexes $^{a}$

\begin{tabular}{|c|c|c|c|c|c|}
\hline \multicolumn{6}{|c|}{$\begin{array}{l}\text { 5'-d(GCGTACXCATGCG)-3' } \\
\text { 3'-d(CGCATḠNGTACGC)-5' }\end{array}$} \\
\hline $\mathbf{X}$ & $\mathbf{N}$ & $T_{\mathrm{m}}\left({ }^{\circ} \mathrm{C}\right)$ & $\mathbf{X}$ & $\mathbf{N}$ & $T_{\mathrm{m}}\left({ }^{\circ} \mathrm{C}\right)$ \\
\hline$d U^{\mathrm{HI}}$ & $\mathrm{dA}$ & 52.1 & $\mathrm{dT}$ & $\mathrm{dA}$ & 57.3 \\
\hline$d U^{F l}$ & $\mathrm{dC}$ & 42.5 & $\mathrm{dT}$ & $\mathrm{dC}$ & 41.2 \\
\hline $\mathbf{d U}^{\mathrm{Fl}}$ & $\mathrm{dG}$ & 45.2 & $\mathrm{dT}$ & $\mathrm{dG}$ & 50.5 \\
\hline$d U^{\mathrm{Fl}}$ & $\mathrm{dT}$ & 47.1 & $\mathrm{dT}$ & $\mathrm{dT}$ & 48.5 \\
\hline
\end{tabular}

${ }^{a}$ Measured at $260 \mathrm{~nm}$ in $10 \mathrm{mM}$ Tris- $\mathrm{HCl}$ buffer (pH 7.2) containing 100 $\mathrm{mM} \mathrm{NaCl}$ and $20 \mathrm{mM} \mathrm{MgCl}_{2}$. Uncertainty in values was less than $0.5^{\circ} \mathrm{C}$.

stable than typical duplexes formed between mispaired $\mathbf{U}^{\mathbf{F l}}$ and natural nucleotide units $(\mathrm{dC}, \mathrm{dG}$, and $\mathrm{dT})$. Thus, despite the only modest stability of the $\mathbf{U}^{\mathrm{Fl}}$ :dA pair, $\mathbf{U}^{\mathrm{F}}$ differentiates strongly between its matched and mismatched nucleobases, participating selectively in a base pair with dA.

We also tested the hybridization properties through fluorescence measurements (Figure 3). First, we measured the fluorescence spectra of the single-stranded ODN 1 and the doublestranded DNAs 1·3-6, each at a concentration of $1.5 \mu \mathrm{M}$ in Tris$\mathrm{HCl}$ buffer (pH 7.2) containing $100 \mathrm{mM} \mathrm{NaCl}$ and $20 \mathrm{mM}$ $\mathrm{MgCl}_{2}$, with excitation at a wavelength of $340 \mathrm{~nm}$. The fluorescence excitation spectra of the single stand and its duplexes exhibited a strong emission peak at $c a$. $430 \mathrm{~nm}$. The fluorescence intensities after hybridization with single base-mismatched targets (ODN 1·ODNs 4-6) were dramatically quenched [7.65- (dC), 5.40- (dG), and 7.07-fold (dT)] relative to that of the singlestrand ODN 1. Based on the relative fluorescence ratios between the fully matched and single base-mismatched duplexes, the fluorescence intensity of the matched duplex $\mathbf{1 \cdot 3}$ was enhanced 11.6-, 8.20-, and 10.7-fold relative to those of the single basemismatched duplexes 1・4-6, respectively. Although these ratios are sufficiently high to allow the recognition of a single base mismatch, our linear beacon system exhibits low detection sen- 


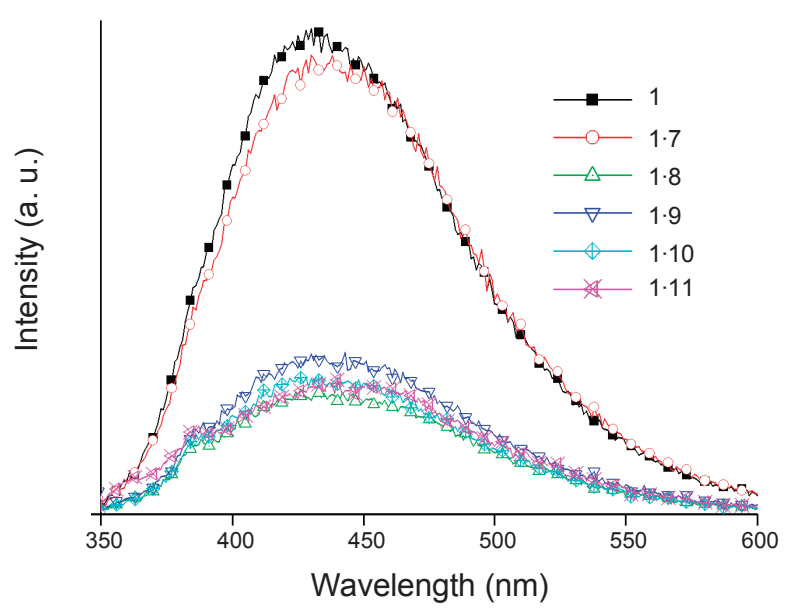

Figure 4. Fluorescence spectra of the single-stranded ODN 1 and the duplexes formed between 1 and the quenchers 7-11. All spectra were recorded at $20^{\circ} \mathrm{C}$ using $1.5 \mu \mathrm{M}$ solutions of the samples in $10 \mathrm{mM}$ Tris$\mathrm{HCl}$ buffer ( $\mathrm{pH} 7.2 ; 100 \mathrm{mM} \mathrm{NaCl}, 20 \mathrm{mM} \mathrm{MgCl}_{2}$ ), with irradiation at $340 \mathrm{~nm}$.

Table 2. Relative fluorescence enhancement ratios upon hybridization between a probe and its targets ${ }^{a}$

\begin{tabular}{cccc}
\hline \multirow{2}{*}{ Probe } & \multicolumn{3}{c}{ Total discrimination factor $^{b}$} \\
\cline { 2 - 4 } & $\mathrm{dA} / \mathrm{dC}$ & $\mathrm{dA} / \mathrm{dG}$ & $\mathrm{dA} / \mathrm{dT}$ \\
\hline $\mathbf{1}$ & 11.6 & 8.20 & 10.7 \\
$\mathbf{1 \cdot 8}$ & 10.1 & 10.6 & 11.2 \\
$\mathbf{1 \cdot 1 1}$ & 12.3 & 10.5 & 11.5 \\
\hline
\end{tabular}

${ }^{a}$ All experiments were conducted using the same concentration $(1.5 \mu \mathrm{M})$ of DNA in $10 \mathrm{mM}$ Tris- $\mathrm{HCl}$ buffer (pH 7.2; $100 \mathrm{mM} \mathrm{NaCl}, 20 \mathrm{mM} \mathrm{MgCl}_{2}$ ), with excitation at $340 \mathrm{~nm} .{ }^{b}$ Relative area ratios of fluorescence intensity ( $350-600 \mathrm{~nm}$ ) between the perfectly matched target 3 and the mismatched targets 4-6, with excitation at $340 \mathrm{~nm}$.

sitivity because of its intrinsically high emission intensity; the fluorescence intensity of the fully matched duplex was enhanced only 1.5 -fold relative to that of single-strand $\mathbf{1}$ (Table 2 ).

To minimize the intrinsic fluorescence of our linear beacon system, we introduced an internal $\mathrm{dG}$ quencher within a strand of fully natural nucleotides; the $\mathrm{dG}$ base was located in the central position, opposite the $\mathbf{U}^{\mathrm{Fl}}$ unit after hybridization with ODN 1. Fluorescence quenching by $\mathrm{dG}$ has been described previously. ${ }^{7-9}$ Because $d G$ has the lowest oxidation potential among the nucleobases, it serves as an intermolecular dynamic quencher nucleotide that operates through photoinduced electron transfer. We synthesized a series of dG-containing ODNs (7-11) as quencher candidates having different nucleotide lengths (from 3 to 11) to determine the optimal quencher length. Thermal denaturation studies of the duplexes formed between ODN 1 and the quenchers 7-11, each at a concentration of $1.5 \mu \mathrm{M}$ in the same aqueous buffer, revealed that only duplex $1 \cdot 11$ exhibited a melting temperature $\left(T_{\mathrm{m}}=34.3^{\circ} \mathrm{C}\right)$. Next, we measured the fluorescence spectra of the hybrids $\mathbf{1 \cdot 7 - 1 1}$ to examine the effect of the quenchers on the fluorescence intensity (Figure 4). Surprisingly, the emission intensity of the linear beacon probe decreased significantly in all cases [3.7- (ODN 8), 2.9- (ODN 9), 3.4-
(ODN 10), and 3.2-fold (ODN 11)], except for the hybrid 1·7, which exhibited no change in fluorescence intensity. This result indicates that the quencher ODN 7 is too short to undergo dynamic hybridization with the linear beacon 1 . Notably, although we would expect ODN 8, with its five-nucleotide sequence, to have very low value of $T_{\mathrm{m}}$, its quenching behavior was as efficient as those of the longer quenchers 9-11. These findings provide strong evidence for ability of $\mathrm{dG}$ to function as a dynamic internal quencher of the fluorescence of ODN 1, and that such a quencher should have more than three nucleotides for effective quenching.

We also examined the effect of the nucleotide context on the fluorescence intensity, positioning mismatched bases (dT instead of dG) on both neighboring sides of the dG unit in strands of different nucleotide lengths, namely 3'-d(TTGTT)-5', 3'-d (ATTGTTA)-5', 3'-d(CATTGTTAC)-5', and 3'-d(GCATTGTTACG)-5' (data not shown). We observed no changes in fluorescence intensity in the presence of mismatched bases located around $\mathrm{dG}$ as a neighboring base. According to our results, improving the ability of quencher requires several conditions to be met: (1) a dG base should be included opposite to the $\mathbf{U}^{\mathrm{Fl}}$ in the sequence to induce dynamic electron transfer; (2) the quencher should feature at least five nucleotides; and (3) matched neighboring bases should be located next to the $\mathrm{dG}$ unit in the quencher sequence.

If a duplex of $\mathbf{U}^{\mathrm{H}}$ quenched with a internal dG quencher would restore its emission intensity as a result of complementary base pairing, but remain in a quenched state upon mismatched hybridization, such a system would be extremely useful for dAspecific SNP typing. To confirm the potential of such an SNP probe, we determined the discriminations toward single-nucleotide modifications displayed by hybrids of the linear beacon probe and internal quenchers (1.8 and 1.11; Figure 5). Upon addition of the perfectly matched target (ODN 3 ) into solutions of both the quenched linear probes $\mathbf{1 \cdot 8}$ and $\mathbf{1 \cdot 1 1}$ (final concentrations of all strands: $1.5 \mu \mathrm{M}$; hybridization time: $5 \mathrm{~min}$ ), the intensity of the fluorescence upon excitation at $340 \mathrm{~nm}$ underwent a 7.6-fold increase for the hybrid $\mathbf{1 \cdot 8}$ and an 8.6-fold increase for the hybrid $\mathbf{1} \cdot \mathbf{1 1}$. In contrast, the formation of mismatched base pairs between $\mathbf{U}^{\mathrm{H}}$ and $\mathrm{dC}, \mathrm{dG}$, and dT units resulted in 0.76- (ODN 4), 0.72- (ODN 5), and 0.68-fold (ODN 6) decreases in fluorescence intensity, respectively, relative to that of the quenched linear probe 1.8 and 0.70-(ODN 4), 0.81-(ODN 5), and 0.75 -fold (ODN 6) decreases in the fluorescence intensity, respectively, relative to that of the quenched linear probe 1.11. Therefore, the total discrimination factors were $10.1(\mathrm{dC})$, $10.6(\mathrm{dG})$, and $11.2(\mathrm{dT})$ when using ODN 8 and $12.3(\mathrm{dC}), 10.5$ (dG), and 11.5 (dT) when using ODN 11 as internal quenchers for the recognition of single base mismatches (Table 2). These values are noteworthy because they suggest that this type of probe would be extremely useful for SNP typing, displaying a highly dA-selective fluorescence emission. Interestingly, we observed only small differences in the total discrimination factors between our previous quencher-free $\mathrm{MB}^{6}$ and our new linear beacon featuring an internal quencher strand. Furthermore, our new linear beacon system provided similar discrimination factors for single base mismatches in the presence of five- and eleven-nucleotide quencher strands, revealing that even a short (five- 
(a)

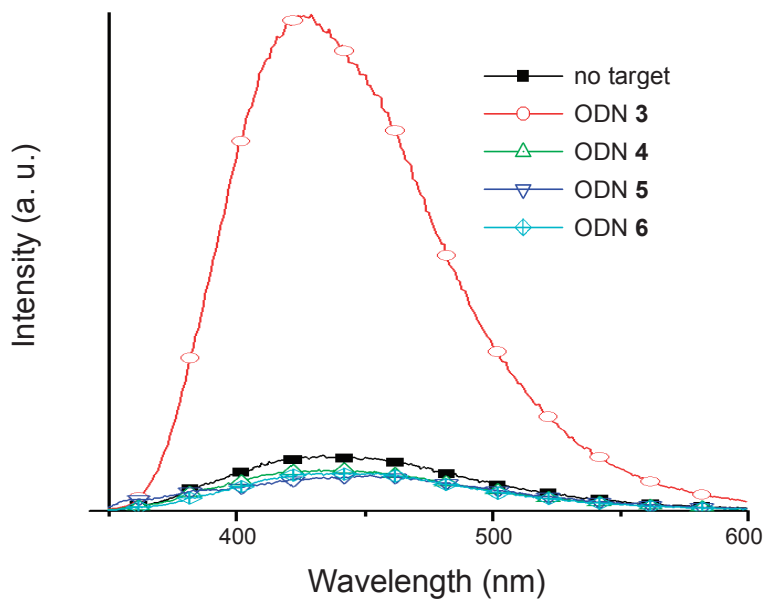

(b)

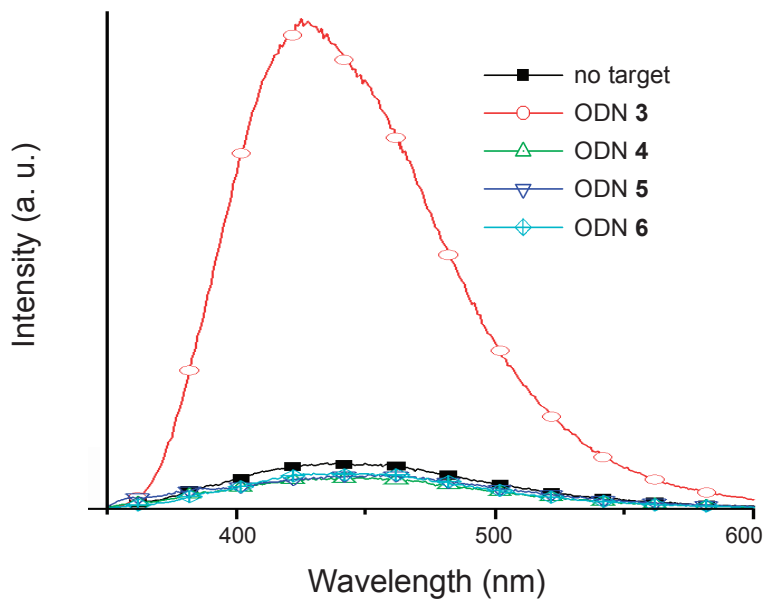

Figure 5. Fluorescence spectra of the duplexes 1.8 (a) and 1.11 (b). All spectra were recorded at $20{ }^{\circ} \mathrm{C}$ using $1.5 \mu \mathrm{M}$ solutions of the samples in $10 \mathrm{mM}$ Tris-HCl buffer (pH 7.2; $100 \mathrm{mM} \mathrm{NaCl}, 20 \mathrm{mM}$ $\mathrm{MgCl}_{2}$ ), with irradiation at $340 \mathrm{~nm}$.

nucleotide) $\mathrm{dG}$ quencher was effective at reducing the intrinsic fluorescence of a linear beacon; because we also observed little effect on the total discrimination factors, our new linear beacon system acts as a highly dA-allele-selective fluorescent "turn on" probe for SNPs.

\section{Conclusion}

We have developed a new base-discriminating linear singlestranded beacon probe, minimizing the fluorescence of the probe itself by introducing dG-containing quencher that functions bas- ed on internal quenching effects. As a result of its reduced intrinsic fluorescence, this probe exhibits a drastic change in fluorescence intensity when it hybridizes with its fully matched target sequence. Furthermore, the total discrimination factors were greater than 10 for all mismatched targets in the presence of even a short (five-nucleotide) dG quencher strand, allowing the recognition of single base mismatches. The synthesis of this linear beacon is relatively simple and inexpensive because no artificial quencher is required; in addition, short nucleotides containing $\mathrm{dG}$ units as internal quenchers are readily prepared inexpensively. Therefore, we expect that this type of beacon system might become a powerful tool for the detection of single nucleotide alterations.

Acknowledgments. This research was supported by Basic Science Research Program through the National Research Foundation of Korea(NRF) funded by the Ministry of Education, Science and Technology(2009-0068091) and the second stage of the Brain Korea 21 Project in 2009.

\section{References}

1. Tyagi, S.; Kramer, F. R. Nat. Biotechnol. 1996, 14, 303. (b) Tyagi, S.; Bratu, D.; Kramer, F. R. Nat. Biotechnol. 1998, 16, 49.

2. Ho, H. A.; Boissinot, M.; Bergeron, M. G.; Corbeil, G.; Dore, K.; Boudreau, D.; Leclerc, M. Angew. Chem., Int. Ed. 2002, 41, 1548. (b) Yamane, A. Nucleic Acids Res. 2002, 30, e97. (c) Kumar, T. S.; Wengel, J.; Hrdlicka, P. J. ChemBioChem 2007, 8, 1122. (d) Thurley, S.; Roglin, L.; Seitz, O. J. Am. Chem. Soc. 2007, 129, 12693.

3. (a) Lyamichev, V.; Mast, A. L.; Hall, J. G.; Prudent, J. R.; Kaiser, M. W.; Takova, T.; Kwiatkowski, R. W.; Sander, T. J.; de Arruda, M.; Arco, D. A.; Neri, B. P.; Brow, M. A. D. Nat. Biotechnol. 1999, 17, 292. (b) Komiyama, M.; Ye, S.; Liang, X. G.; Yamamoto, Y.; Tomita, T.; Zhou, J. M.; Aburatani, H. J. Am. Chem. Soc. 2003, 125,3758 .

4. (a) Schena, M.; Heller, R. A.; Theriault, T. P.; Konrad, K.; Lachenmeier, E.; Davis, R. W. Trends Biotechnol. 1998, 16, 301. (b) Pirrung, M. C. Angew. Chem., Int. Ed. 2002, 41, 1276.

5. (a) Taton, T. A.; Mirkin, C. A.; Letsinger, R. L. Science 2000, 289, 1757; (b) Liu, G.; Lee, T. M. H.; Wang, J. J. Am. Chem. Soc. 2005, 127,38 .

6. Hwang, G. T.; Seo, Y. J.; Kim, B. H. J. Am. Chem. Soc. 2004, 126, 6528.

7. Luo, G.; Zheng, L.; Zhang, X.; Zhang, J.; Nilsson-Ehle, P.; Xu, N. Anal. Biochem. 2009, 386, 161.

8. Seidel, C. A. M.; Schulz, A.; Sauer, M. H. M. J. Phys. Chem. 1996, 100,5541 .

9. Cooper, J. P.; Hagerman, P. J. Biochemistry 1990, $29,9261$.

10. Gait, M. J. Oligonucleotide Synthesis: A Practical Approach; IRL Press: Washington, DC, 1984.

11. Ryu, J. H.; Seo, Y. J.; Hwang, G. T.; Lee, J. Y.; Kim, B. H. Tetrahedron 2007, 63, 3538.

12. Wagenknecht, H.-A. Angew. Chem., Int. Ed. 2003, 42, 2454. 